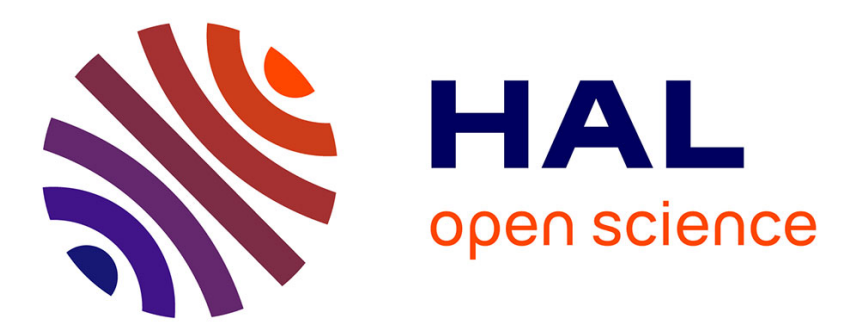

\title{
An EM algorithm for path delay and complex gain estimation of slowly varying fading channel for CPM signals
}

\author{
Habti Abeida, Jean-Marc Brossier, Laurent Ros, Jordi Vilà Valls
}

\section{- To cite this version:}

Habti Abeida, Jean-Marc Brossier, Laurent Ros, Jordi Vilà Valls. An EM algorithm for path delay and complex gain estimation of slowly varying fading channel for CPM signals. Globecom 2009 - IEEE Global Communication Conference (Globecom'09), Nov 2009, Honolulu, Hawaii, United States. 6 p. hal-00447404

\author{
HAL Id: hal-00447404 \\ https://hal.science/hal-00447404
}

Submitted on 14 Jan 2010

HAL is a multi-disciplinary open access archive for the deposit and dissemination of scientific research documents, whether they are published or not. The documents may come from teaching and research institutions in France or abroad, or from public or private research centers.
L'archive ouverte pluridisciplinaire HAL, est destinée au dépôt et à la diffusion de documents scientifiques de niveau recherche, publiés ou non, émanant des établissements d'enseignement et de recherche français ou étrangers, des laboratoires publics ou privés. 


\title{
AN EM ALGORITHM FOR PATH DELAY AND COMPLEX GAIN ESTIMATION OF SLOWLY VARYING FADING CHANNEL FOR CPM SIGNALS
}

\author{
H. Abeida, J.-M. Brossier, L. Ros and J. Vilà Valls \\ GIPSA-lab/DIS - BP 46 - 38402 Saint-Martin-d'Hères - France
}

\begin{abstract}
This paper addresses the joint path delay and time-varying complex gain estimation for continuous phase modulation $(\mathrm{C}$ PM) over a time-selective slowly varying flat Rayleigh fading channel. We propose an expectation-maximization (EM) algorithm for path delay estimation in a Kalman smoother framework. The time-varying complex gain is modeled by a first order autoregressive (AR) process. Such a modeling yields to the representation of the problem by a dynamic bayesian system in a state-space form that allows the application of EM algorithm in the context of unobserved data for obtaining an estimate of the path delay. This is used with Kalman smoother for state estimation. We derive analytically a closedform expression of the modified hybrid Cramér-Rao bound (MHCRB) for path delay and complex gain parameters. Finally, some numerical examples are presented to illustrate the performance of the proposed algorithm compared to the conventional generalized correlation method and to the MHCRB.
\end{abstract}

Index Terms - CPM signal, EM algorithm, hybrid Cramér Rao bound, path delay estimation, maximum-likelihood (ML) estimation, Kalman smoother filter, Kalman filter, fading channels.

\section{INTRODUCTION}

Continuous phase modulation (CPM) is preferred in numerous wireless communications and mobile applications for its constant envelope property and high spectral efficiency [1]. Binary CPM systems, such as minimum-shift keying (MSK) and Gaussian MSK (GMSK), containing non-circular (or improper) process $[2,3]$, have been widely employed in many applications.

Due to the importance of CPM signals, many frequency and timing synchronization algorithms have been developed for such signals $[4,5]$. These algorithms, typically categorized in Data-Aided (DA) (see, e.g. [6]) and Non-Data-Aided (NDA) (see, e.g. [7, 8, 9]) methods, have been designed under the assumption of additive white Gaussian noise channel (AWGN). However, few research works address CPM time synchronization over a time-varying channel. We can cite the recent works proposed in $[13,18]$ for flat-fading channels. A maximum likelihood (ML) approach was employed in [13] for estimating time delay for CPM signals in the special case of MSK signal. However, [13, 18] do not consider the bayesian approach to take into account the prior distribution of the unknown time-varying complex gains of the channel, which need to be estimated in practice.

Assuming that the time-varying complex gains can be modeled by a first order autoregressive (AR1) process (e.g. [22]), the problem of time synchronization over Rayleigh flat-fading channels can be formulated as a dynamic state-space bayesian system with unknown (hidden) complex gains. So, we are facing a problem of state estimation in a nonlinear dynamical system with unknown parameters, which is a problem of practical interest in numerous applications. In many cases, the parameters of the dynamic model for a real system are not known exactly and need to be estimated. In reference [14], an EM algorithm [12] combined with a Kalman smoother [23] was proposed to compute the ML estimates of the speech recognition system parameters while also providing the state estimates. Recently, the EM algorithm has been applied to a lot of problems for parameter estimation and learning (see e.g. $[15,17,16])$.

In order to evaluate the estimator performance, lower bounds on the Mean Square Error (MSE) are needed. One of the most used is the Cramér-Rao Bound (CRB) [29]. Depending on the prior knowledge available on parameters, the CRB has different expressions. Among which, the Hybrid CRB (HCRB) is considered in the case of hybrid vector that contains deterministic and random parameters (see e.g., [26, 27]). We note that the HCRB generalizes the classical CRB (see e.g., [29, 28]) and the bayesian CRB (BCRB) [24]. However, the true expression of CRB is sometimes difficult to derive analytically. To overcome this difficulty, other CRB's have been considered in the literature such as the Modified CRB (MCRB) (see, e.g., [4]), which is in general looser (i.e., lower) than the CRB.

In this paper, we describe the EM algorithm for jointly estimating path delay and complex gains over a slow Rayleigh flat-fading channel in the general case of a CPM signal. We first model the time-varying complex gains with a state space model. Then, we estimate the path delay with an EM algorithm. Once we have the estimate of the path delay, complex gains estimates are computed with Kalman smoother. We also derive an analytical closed-form expression of the modified HCRB (MHCRB) for path delay and complex gains. This 
bound is used to evaluate the performance of the proposed EM algorithm.

The paper is organized as follows. Section 2 describes the CPM signal model, the AR model and the state-space representation of the problem. The EM algorithm is presented in section 3. The MHCRB is derived in section 4 and finally some simulations are presented in section 5 .

The following notations are used throughout the paper. Matrices and vectors are represented by bold upper case and bold lower case characters, respectively. I is the identity matrix. Vectors are by default in column orientation, while $T, H$ and $*$ stand for transpose, conjugate transpose and conjugate, respectively. $\mathrm{E}(),. \operatorname{Tr}($.$) and \|$.$\| are the expectation, trace and$ norm operators respectively.

\section{PROBLEM STATEMENT AND ESTIMATION OBJECTIVES}

Following [1], the complex envelope $s(t, \mathbf{a})$ of a CPM signal can be written as $s(t, \mathbf{a})=e^{i \phi(t, \mathbf{a})}$ where the phase $\phi(t, \mathbf{a})$ of $s(t, \mathbf{a})$ is given by

$$
\phi(t, \mathbf{a})=2 \pi h \sum_{j \in \mathbb{Z}} a_{j} q(t-j T)
$$

where $T$ is the symbol period and $\mathbf{a} \stackrel{\text { def }}{=}\left(\ldots, a_{-2}, a_{-1}, a_{0}, a_{1}\right.$ $\left., a_{2}, \ldots\right)$ is the independent identically distributed (i.i.d.) binary data sequence, with each element taking on values $\{ \pm 1\}$. $q(t)=\int_{0}^{t} g(u) d u$ corresponds to the phase pulse shaping function that describes how the underlying phase change $\pm \pi h$ evolves with time where $g(t)$, the frequency shaping filter, is positive and non-zero on the interval $[0, L T], L$ is the correlation length. The modulation index $h$ determines the rate of change of frequency in the signalling interval. Finally, we note that if $n T \leq t \leq(n+1) T$ the phase $\phi(t, \mathbf{a})$ given by (1) can be written as

$$
\phi(t, \mathbf{a})=\pi h \sum_{j=-\infty}^{n-L} a_{j}+2 \pi h \sum_{j=0}^{L-1} a_{n-j} q(t-(n-j) T) .
$$

Transmitting $s(t, \mathbf{a})$ over a frequency-flat, slow fading Rayleigh channel results in the following received waveform

$$
y(t)=\alpha(t) s(t-\tau, \mathbf{a})+b(t),
$$

where $\alpha(t)$ is a zero-mean Gaussian complex circular multiplicative gain of variance $\sigma_{\alpha}^{2}$, introduced by the flat fading channel with autocorrelation function $R_{\alpha}(\Delta t) \stackrel{\text { def }}{=} \sigma_{\alpha}^{2} \mathrm{E}(\alpha(t)$ $\left.\alpha^{*}(t-\Delta t)\right), \tau$ is the fixed unknown path delay, and $b(t)$ is an additive white Gaussian complex circular noise with bilateral spectral power density $N_{0}$.

The equivalent discrete-time signal model observed during $N$ signaling intervals, after low-pass filtering and sampling at rate $T_{s}=T / M$ is given by

$$
y\left(k T_{s}\right)=\alpha\left(k T_{s}\right) s_{k}(\tau, \mathbf{a})+b\left(k T_{s}\right), k=0, \ldots, M N-1
$$

where $s_{k}(\tau, \mathbf{a}) \stackrel{\text { def }}{=} s\left(k T_{s}-\tau, \mathbf{a}\right)=e^{i \phi_{k}(\tau, \mathbf{a})}$ and $\phi_{k}(\tau, \mathbf{a}) \stackrel{\text { def }}{=}$ $\phi\left(k T_{s}-\tau, \mathbf{a}\right)$. Note that, after antialiasing filtering (with cutoff frequency $M / T$ ) and sampling, the noise term $b(k)$ is assumed white with a known variance $\sigma^{2}=\frac{N_{0}}{T_{s}}$.

From (2), we obtain after some easy manipulations of indices that the phase term $\phi_{k}(\tau, \mathbf{a})$ can be expressed as

$$
\begin{aligned}
\phi_{n M+m}(\tau, \mathbf{a}) & \stackrel{\text { def }}{=} \phi\left((n M+m) T_{s}-\tau, \mathbf{a}\right) \\
& =\pi h \sum_{j=-\infty}^{n-L} a_{j}+2 \pi h \sum_{j=0}^{L-1} a_{n-j} q_{j, m}(\tau),(5)
\end{aligned}
$$

for each $n \geq 0$ and for each $m$ such as $0 \leq m \leq M-1$, the $\tau$-dependent coefficients $q_{j, m}(\tau)$ are defined by $q_{j, m}(\tau) \stackrel{\text { def }}{=}$ $q\left(m T_{s}+j T-\tau\right)$. The complex gain of the channel $\alpha($.) does not change during symbol period but varies from symbol to symbol because the gain is assumed to be slowly timevarying. This implies that the coefficients $\alpha\left((n M+m) T_{s}\right)$ for $m=0, \ldots, M-1$, all equal to the same value denoted by $\alpha_{n}$. Then the discrete-time version of (4) can be written as follows:

$$
y\left((n M+m) T_{s}\right)=\alpha_{n} s_{n M+m}(\tau, \mathbf{a})+b\left((n M+m) T_{s}\right) .
$$

Collecting the samples of the received signal within one slot to form a vector $\mathbf{y}_{n} \stackrel{\text { def }}{=}\left(y\left((n M) T_{s}\right), \ldots, y((n M+M-\right.$ 1) $\left.\left.T_{s}\right)\right)^{T}$ yields the following model

$$
\mathbf{y}_{n}=\alpha_{n} \mathbf{g}_{n}(\tau, \mathbf{a})+\mathbf{b}_{n},
$$

where $\mathbf{g}_{n}(\tau, \mathbf{a}) \stackrel{\text { def }}{=}\left(e^{i \phi_{n M}(\tau, \mathbf{a})}, \ldots, e^{i \phi_{n M+M-1}(\tau, \mathbf{a})}\right)^{T}$, and $\mathbf{b}_{n} \stackrel{\text { def }}{=}\left(b\left((n M) T_{s}\right), \ldots, b\left((n M+M-1) T_{s}\right)\right)^{T}$ is a $M \times 1$ noise vector with covariance matrix $\sigma^{2} \mathbf{I}$.

Among various channel models, the information theoretic results in [10] show that the first-order AR model provides a sufficiently accurate model for time-selective fading channels and therefore, will be adapted henceforth. Specifically, $\alpha_{n}$ varies according to

$$
\alpha_{n}=\gamma \alpha_{n-1}+e_{n}
$$

where the noise $e_{n}$ is zero-mean Gaussian complex circular with a known variance $\sigma_{e}^{2}$ and is statistically independent of $\alpha_{n-1}$. Using (8), simple manipulations lead to

$$
\sigma_{e}^{2}=\sigma_{\alpha}^{2}\left(1-\gamma^{2}\right) \text { and } \gamma=\mathrm{E}\left(\alpha_{n} \alpha_{n-1}^{*}\right)
$$

According to Jakes' model [21], we have $\gamma=J_{0}\left(2 \pi f_{d} T\right)$, where $J_{0}($.$) is the first kind 0th-order Bessel function and f_{d}$ denotes the maximum Doppler shift.

Having the model for the variation of the channel, and from eq. (7), we can obtain the following state space representation of the problem

$$
\left\{\begin{array}{l}
\alpha_{n}=\gamma \alpha_{n-1}+e_{n} \\
\mathbf{y}_{n}=\alpha_{n} \mathbf{g}_{n}(\tau, \mathbf{a})+\mathbf{b}_{n}
\end{array}\right.
$$


The initial state $\alpha_{0}$ is assumed to be Gaussian complex circular with a known variance $\sigma_{0}^{2}$.

In general, the objective is to jointly estimate the path delay parameter $\tau$ and the state $\boldsymbol{\alpha} \stackrel{\text { def }}{=}\left(\alpha_{1}, \ldots, \alpha_{N-1}\right)$ using the set of received signals $\mathbf{y} \stackrel{\text { def }}{=}\left(\mathbf{y}_{0}^{T}, \ldots, \mathbf{y}_{N-1}^{T}\right)^{T}$. In this paper, however, we will assume, except in section 4 , that the transmit symbol sequence $\mathbf{a}$ is known at the receiver, and in order to simplify notation we use $\mathbf{g}_{n}(\tau) \stackrel{\text { def }}{=} \mathbf{g}_{n}(\tau, \mathbf{a})$.

Note that if $\tau$ is known, the state parameters $\alpha_{n}$ can be inferred using a Kalman smoother [11]. We note that, due to the presence of unobserved data $\boldsymbol{\alpha}$, the maximum likelihood (ML) method can not be used because the computation of the likelihood function $f(\mathbf{y} \mid \mathbf{a} ; \tau)=\mathrm{E}(f(\mathbf{y} \mid \boldsymbol{\alpha}, \mathbf{a} ; \tau))$ in a closed-form and its maximization w.r.t. $\tau$ seems to be an intractable problem. In the following section, we describe the Expectation-Maximization (EM) algorithm to find the ML estimates.

\section{THE EM ALGORITHM}

The EM algorithm [12] is an iterative method to find the ML estimates of parameters in the presence of unobserved data. The idea behind the algorithm is to augment the observed data with latent data, which can be either missing data or parameter values. The algorithm can be broken down into two steps: the E-step and the M-step. We now describe an EM algorithm for our model. Following the procedure given in [14, Sec. B], we consider the received data $\mathbf{y}$ as incomplete data, and define the complete data as $\mathbf{z} \stackrel{\text { def }}{=}\left(\mathbf{y}^{T}, \boldsymbol{\alpha}^{T}\right)^{T}$. Since the state is Markov, the likelihood function of the complete data is given by

$$
P(\mathbf{z} \mid \mathbf{a} ; \tau)=P\left(\alpha_{0}\right) \prod_{n=1}^{N-1} P\left(\alpha_{n} \mid \alpha_{n-1}\right) \prod_{n=0}^{N-1} P\left(\mathbf{y}_{n} \mid \boldsymbol{\alpha}_{n}, \mathbf{a} ; \tau\right)
$$

Due to the Gaussian noise assumption, we have

$$
\begin{aligned}
\ln (P(\mathbf{z} ; \tau)) & =C-\frac{1}{\sigma^{2}} \sum_{n=0}^{N-1}\left\|\mathbf{y}_{n}-\alpha_{n} \mathbf{g}_{n}(\tau)\right\|^{2} \\
& -\frac{1}{\sigma_{e}^{2}} \sum_{n=1}^{N-1}\left|\alpha_{n}-\gamma \alpha_{n-1}\right|^{2}-\frac{1}{\sigma_{0}^{2}}\left\|\alpha_{0}\right\|^{2}
\end{aligned}
$$

where $C$ is a constant that only depends on the state noise variances. Each iterative process $p=0,1,2, \ldots$, in the EM algorithm for estimating $\tau$ from $\mathbf{y}$ consists of the following two steps:

E-step. Given the measurements $\mathbf{y}$ and an estimate of the model parameter from the previous iteration $\tau^{(p)}$, we calculate:

$$
\mathrm{Q}\left(\tau, \tau^{(p)}\right) \stackrel{\text { def }}{=} \mathrm{E}\left(\ln P(\mathbf{z} ; \tau) \mid \mathbf{y}, \mathbf{a} ; \tau^{(p)}\right),
$$

where the expectation is taken with respect to $\boldsymbol{\alpha}$ conditioned on $\mathbf{y}$ and the latest estimate of $\tau, \tau^{(p)}$.
M-step. This step finds $\boldsymbol{\tau}^{(p+1)}$, the value of $\tau$ that maximizes $\mathrm{Q}\left(\tau, \tau^{(p)}\right)$ over all possible values of $\tau$ :

$$
\boldsymbol{\tau}^{(p+1)}=\operatorname{argmax}_{\tau} Q\left(\tau, \tau^{(p)}\right)
$$

This procedure is repeated until the sequences $\tau^{(0)}, \tau^{(1)}$, ... converges.

The E-step uses a Kalman smoother to estimate the state $\alpha_{n}$ for which the function $\mathrm{Q}$ can be expressed as (see Appendix A) :

$$
\begin{aligned}
\mathrm{Q}\left(\tau, \tau^{(p)}\right) & =-\frac{1}{\sigma^{2}} \sum_{n=1}^{N}\left(\operatorname { T r } \left(S_{n \mid N}^{(p)} \mathbf{g}_{n}(\tau) \mathbf{g}_{n}^{H}(\tau)\right.\right. \\
& \left.\left.+\left(\mathbf{y}_{n}-\hat{\alpha}_{n \mid N}^{(p)} \mathbf{g}_{n}(\tau)\right)\left(\mathbf{y}_{n}-\hat{\alpha}_{n \mid N}^{(p)} \mathbf{g}_{n}(\tau)\right)^{H}\right)\right)
\end{aligned}
$$

where $\hat{\alpha}_{n \mid N}^{(p)} \stackrel{\text { def }}{=} \mathrm{E}\left(\alpha_{n} \mid \mathbf{y}, \mathbf{a} ; \tau^{(p)}\right)$ and $S_{n \mid N}^{(p)} \stackrel{\text { def }}{=} \mathrm{E}\left(\left(\alpha_{n}-\right.\right.$ $\left.\left.\hat{\alpha}_{n \mid N}^{(p)}\right)\left(\alpha_{n}-\hat{\alpha}_{n \mid N}^{(p)}\right)^{H} \mid \mathbf{y}, \mathbf{a} ; \tau^{(p)}\right)$ can be computed for all $n=$ $0, \ldots, N-1$ from the fixed interval Kalman smoother [14, 23], using the parameter estimates obtained at iteration $p$. The smoother consists of a Backward pass that follows the standard Kalman filter Forward recursions given as:

Forward recursion:

$$
\begin{aligned}
\hat{\alpha}_{n+1 \mid n}^{(p)} & =\gamma \hat{\alpha}_{n}^{(p)} \\
S_{n+1 \mid n}^{(p)} & =\gamma^{2} S_{n}^{(p)}+\sigma_{e}^{2} \\
K_{n+1} & =S_{n+1 \mid n}^{(p)} \mathbf{g}_{n}^{H}\left(\tau^{(p)}\right)\left(\sigma^{2} \mathbf{I}+S_{n+1 \mid n}^{(p)} \mathbf{g}_{n}\left(\tau^{(p)}\right) \mathbf{g}_{n}^{H}\left(\tau^{(p)}\right)\right) \\
\hat{\alpha}_{n+1}^{(p)} & =\hat{\alpha}_{n+1 \mid n}^{(p)}+K_{n+1}\left(\mathbf{y}_{n}-\mathbf{g}_{n}\left(\tau^{(p)}\right) \hat{\alpha}_{n+1 \mid n}^{(p)}\right) \\
S_{n+1}^{(p)} & =S_{n+1 \mid n}^{(p)}-S_{n+1 \mid n}^{(p)} K_{n+1} \mathbf{g}_{n}\left(\tau^{(p)}\right)
\end{aligned}
$$

Backward recursion:

$$
\begin{aligned}
J_{n-1} & =\gamma S_{n-1}^{(p)} S_{n \mid n-1}^{(p)-1} \\
\hat{\alpha}_{n-1 \mid N}^{(p)} & =\hat{\alpha}_{n-1}^{(p)} J_{n-1}\left(\hat{\alpha}_{n \mid N}^{(p)}-\gamma \hat{\alpha}_{n-1}^{(p)}\right) \\
S_{n-1 \mid N}^{(p)} & =S_{n-1}^{(p)}+J_{n-1}\left(S_{n \mid N}^{(p)}-S_{n \mid n-1}^{(p)}\right) J_{n-1}^{*} \\
S_{n, n-1 \mid N}^{(p)} & =S_{n}^{(p)} J_{n-1}^{H}+J_{n}\left(S_{n+1, n \mid N}^{(p)}-\gamma S_{n}^{(p)}\right) J_{n-1}^{*}
\end{aligned}
$$

Remark 1 We note that the steps of the EM algorithm can be extended in the case where the parameters $\left(\sigma^{2}, \sigma_{e}^{2}, \sigma_{0}^{2}, \gamma\right)$ are assumed unknown. By adapting the same steps of the approach proposed in [15], the estimates of these parameters can be obtained in E-step by maximizing the function $\mathrm{Q}$ given by (18).

\section{HYBRID CRAMÉR RAO BOUND}

The CRB is an important criterion to evaluate how good any unbiased estimator can be since it provides the MSE bound among all unbiased estimators. In this section we assume that 
the symbol $\left\{a_{n}\right\}$ are i.i.d. and equiprobable with each element taking on values $\{ \pm 1\}$. Since the parameters of interest are the deterministic parameter $\tau$ and the random parameter state $\boldsymbol{\alpha}$, we have derived in [25] an analytical expression of the MHCRB using well known properties of the gaussian distribution and Markov state evolution of AR parameters. In Section 5, we will show the performance of the proposed EM algorithm and compare it to the MHCRB.

Result 1 The state and delay parameters are decoupled in the modified hybrid Fisher information matrix (MHFIM) in the case of CPM signals as follows:

$$
\mathbf{I}=\left(\begin{array}{cc}
I_{(\tau, \tau)} & \mathbf{0} \\
\mathbf{0} & \mathbf{I}_{(\alpha, \alpha)}+\mathbf{B}
\end{array}\right)
$$

where $I_{(\tau, \tau)}=8 \pi^{2} h^{2} N \rho \xi(\tau), \mathbf{I}_{(\alpha, \alpha)}=\frac{M}{\sigma^{2}} \mathbf{I}$ and the matrix $\mathbf{B}$ has the following non-zeros elements $\mathbf{B}(1,1)=\frac{1+\gamma^{2}}{\sigma_{e}^{2}}-$ $\mathrm{E}_{\alpha_{0}}\left(\frac{\partial^{2} \ln P\left(\alpha_{0}\right)}{\partial \alpha_{0} \partial \alpha_{0}^{*}}\right), \mathbf{B}(k, k)=\frac{1+\gamma^{2}}{\sigma_{e}^{2}}$ and $\mathbf{B}(k, k-1)=\mathbf{B}(k-$ $1, k)=-\frac{\gamma}{\sigma_{e}^{2}}$, for $k=2, \ldots, N$, and where $\rho \stackrel{\text { def }}{=} \frac{\sigma_{\alpha}^{2}}{\sigma^{2}}$ is the $S N R$ and $\xi(\tau) \stackrel{\text { def }}{=} \sum_{m=0}^{M-1} \sum_{j=0}^{L-1} g^{2}\left(m T_{e}+j T-\tau\right)$. Consequently

$$
\begin{aligned}
\operatorname{MHCRB}(\tau) & =\frac{1}{8 \pi^{2} h^{2} N \xi(\tau)} \frac{1}{\rho} \\
\operatorname{MHCRB}(\boldsymbol{\alpha}) & =\left(\frac{M}{\sigma^{2}}+\mathbf{B}\right)^{-1}
\end{aligned}
$$

We assume a classical non-informative prior on $\alpha_{0}$ (see, e.g., [19]). As a consequence, $\mathrm{E}_{\alpha_{0}}\left(\frac{\partial^{2} \ln P\left(\alpha_{0}\right)}{\partial \alpha_{0} \partial \alpha_{0}^{*}}\right)=0$.

We remark that the $\operatorname{MHCRB}(\tau)$ is inversely proportional to $\rho$ and depends on the modulation index $h$, the shaping filter $g$ and the correlation length. (16) remains valid for all CPM signal. This expression also is similar to the MCRB derived in $[4$, rel. (2.4.54)]. Finally, we remark that the $\operatorname{MHCRB}(\boldsymbol{\alpha})$ does not depend on the parameter $\tau$.

\section{SIMULATION RESULTS}

In this section, we present numerical examples to illustrate the performance of the proposed algorithm to estimate jointly the path delay and complex gains in the special case of binary GMSK signal with a bandwidth-bit time product $B T=0.3$, a modulation index $h=\frac{1}{2}$ and a $4 T$-wide approximation of the Gaussian shaping filter, i.e. $L=4$ (see e.g. [4, rel. (4.2.8)]). These parameters are those of GSM systems. The channel is simulated according to the Jakes model [21,22] with dopplertime product of $f_{d} T=0.000738$, corresponding to a carrier frequency of $1.8 \mathrm{GHz}$, a mobile speed of $120 \mathrm{~km} / \mathrm{h}$, and a transmission rate of $270 \mathrm{~kb} / \mathrm{s}$. First order AR process, with a known coefficient $\gamma=0.99999$ (which corresponds to a slow fading channel) is chosen to model the time variation of the complex gains of the channel. The symbols $\left\{a_{n}\right\}$ are assumed known at the receiver, the number $N$ of signaling intervals is set to $N=200$, the oversampling ratio is equal to $M=8$, and a fixed value $(\tau=0.4)$ is used as the normalized unknown delay $\tau / T$. In the simulations, each value of the MSE is obtained by averaging over 1000 independent runs.

The initial estimate of the unknown parameter $\tau$ is given by the correlation method or chosen in the vicinity of $0.4 T$, and the channel is initialized to the known state $\alpha_{0}$ assumed to be a trial of a complex Gaussian random variable with a known variance $\sigma_{0}^{2}=1$.

Fig. 1 shows one realization of the recursive estimates of the normalized path delay obtained with the EM algorithm versus the number of iterations for a SNR of $30 \mathrm{~dB}$. This figure shows the estimated normalized path delay parameter converges to the true value fairly quickly in the case of slow flat-fading channel. We note that the EM algorithm still gives a valid estimate of $\tau$ when $f_{d} T>0.001$; however, in this case, the EM algorithm converges after about 30 iterations as shown in this figure with $f_{d} T=0.02$.

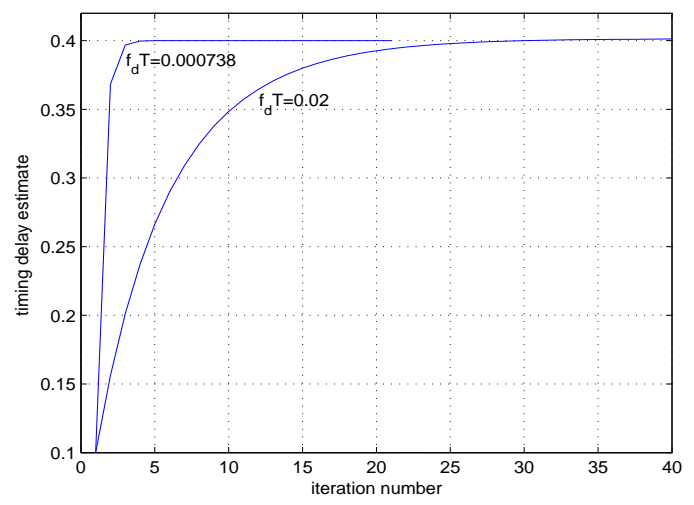

Fig.1 An EM trajectory for two values of $f_{d} T$ with $S N R=30 \mathrm{~dB}$.

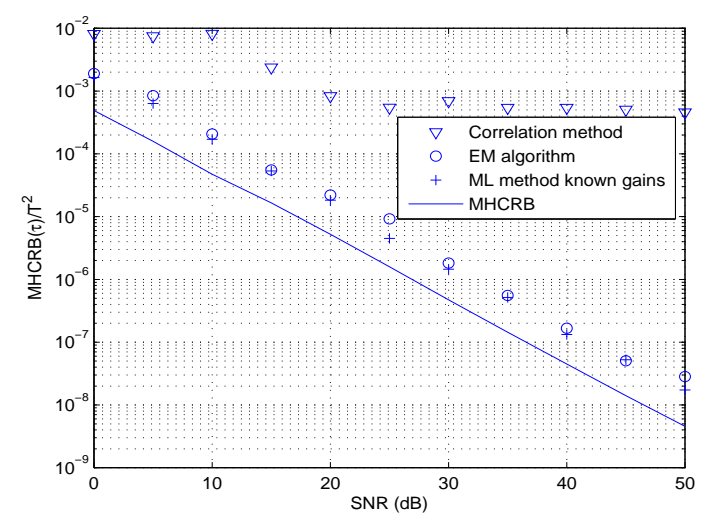

Fig.2 Normalized $\operatorname{MHCRB}(\tau) / T^{2}$, and estimated $\operatorname{MSE} \mathrm{E}(\hat{\tau}-\tau)^{2} / T^{2}$ given by the EM algorithm (ten iterations) and by the correlation method for $f_{d} T=0.000738$, versus SNR.

Fig. 2 compares the $\operatorname{MHCRB}(\tau)$ normalized to $T^{2}$ (given by (16)) to the MSE of the normalized path delay (i.e. $\mathrm{E}(\hat{\tau}-$ $\tau)^{2} / T^{2}$ ) given by the EM algorithm initialized by the estimate given by the correlation method (see, e.g. [20]), and the 
correlation method, as a function of the SNR. For comparison purpose, we have computed the MSE associated to the ML method with a perfect knowledge of the complex gains. As seen from the shown simulation results, performance of the EM algorithm is very close to the ML method in the case of perfect knowledge of the complex gains. We observe also that the EM algorithm significantly outperforms the correlation method based on the maximum of the delay-Doppler ambiguity function. On the other hand, the performance of the $\mathrm{EM}$ algorithm is close to the MHCRB contrarily to those of the correlation method.

Fig. 3 compares the MSE for the complex gains $\mathrm{E}(\| \hat{\boldsymbol{\alpha}}-$ $\left.\boldsymbol{\alpha} \|^{2}\right)$ given by the EM algorithm, the Kalman smoother with a perfect knowledge of the delay path to the $\operatorname{MHCRB}(\boldsymbol{\alpha})$ given by (17), as a function of SNR. We see that the performance of the EM algorithm and the Kalman smoother are very close, and the associated estimates reach the MHCRB when the SNR increases. At low SNR, We recall that the Modified CRB is, in general, looser than the true CRB.

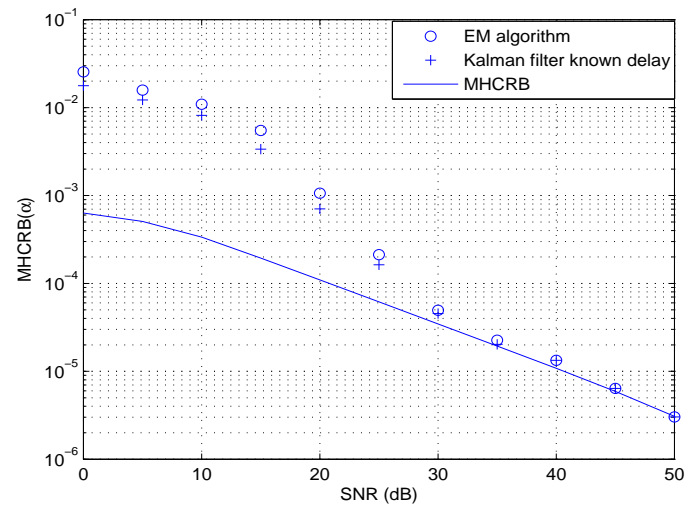

Fig.3 Normalized MHCRB $(\boldsymbol{\alpha})$, and estimated normalized MSE $\frac{1}{N} \mathrm{E}(\| \hat{\boldsymbol{\alpha}}-$ $\boldsymbol{\alpha} \|^{2}$ ) given by the EM algorithm (ten iterations) for $f_{d} T=0.000738$, versus SNR.

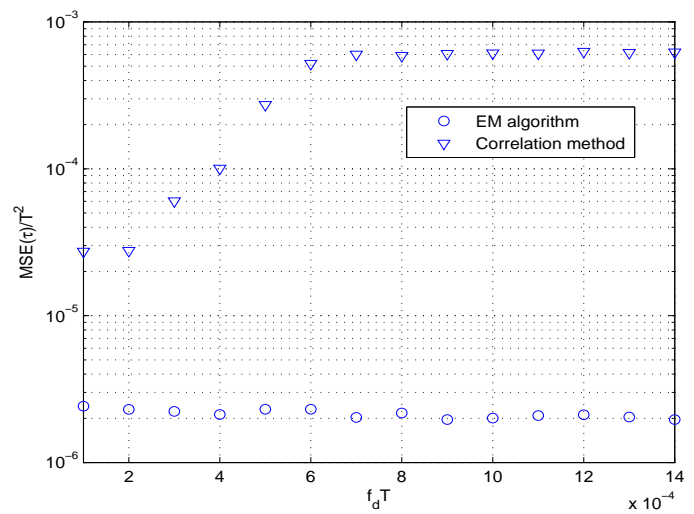

Fig.4 The normalized MSE $\mathrm{E}(\hat{\tau}-\tau)^{2} / T^{2}$ given by the EM algorithm (ten iterations) and by the correlation method for $S N R=30 \mathrm{~dB}$, versus $f_{d} T$.

Fig 4 compares the normalized MSE $\mathrm{E}(\hat{\tau}-\tau)^{2} / T^{2}$ given by the EM algorithm and the correlation method (see, e.g. [20]), as a function of $f_{d} T$ with $S N R=30 \mathrm{~dB}$. We observe from this figure that as $f_{d} T$ increases, the MSE of the delay given by the EM algorithm remains almost constant contrarily to the MSE given by the correlation method which increases when $f_{d} T$ increases.

\section{CONCLUSION}

We have presented an EM algorithm for joint path delay and time-varying complex gains estimation for CPM signals over a time-selective slowly varying flat Rayleigh fading channel. We have modeled the flat fading channel as a first order autoregressive process. The EM algorithm has been combined with Kalman smoother to yield time-varying complex gains estimation and ML estimate of the path delay. The proposed algorithm was reduced to a single-parameter search over the path delay only. We have also derived a closed-form expression of the MHCRB for path delay and time-varying complex gains parameters. The performance of the proposed algorithm have been evaluated in terms of the MSE and the MHCRB. Finally, the simulation results have shown that the proposed algorithm provides better estimation of the delay and complex gains parameters compared to the conventional correlation method. Moreover, the performance of the proposed algorithm in term of delay estimation is very close to the performance of the ML method in the case of perfect knowledge of complex gains.

\section{Acknowledgement}

This work was supported in part by the French ANR (Agence Nationale de la Recherche), LURGA project, and by the COST Action IC0803 RF/Microwave Communication Subsystems for Emerging Wireless Technologies (RFCSET)

\section{A. APPENDIX: PROOF OF REL. (13)}

Taking the expectation with respect to $\boldsymbol{\alpha}$ conditioned on $\mathbf{y}$, given the current parameter estimate $\tau^{(p)}$, we obtain from (12) the expectation of the log-likelihood function of the complete data which can be expressed as

$$
\begin{aligned}
& \mathrm{Q}\left(\tau, \tau^{(p)}\right)=\mathrm{E}\left(\ln \left(P(\mathbf{z} ; \tau) \mid \mathbf{y}, \mathbf{a} ; \tau^{(p)}\right)\right. \\
= & c\left(\sigma^{2}, \sigma_{e}^{2}, \sigma_{0}^{2}\right)-\frac{1}{\sigma^{2}} \sum_{n=0}^{N-1} \operatorname{Tr}\left(\mathbf{y}_{n} \mathbf{y}_{n}^{H}\right. \\
+ & \left.P_{n \mid N}^{(p)} \mathbf{g}_{n}(\tau) \mathbf{g}_{n}^{H}(\tau)-\hat{\alpha}_{n \mid N}^{(p) *} \mathbf{y}_{n} \mathbf{g}_{n}^{H}(\tau)-\hat{\alpha}_{n \mid N}^{(p)} \mathbf{g}_{n}(\tau) \mathbf{y}_{n}^{H}\right) \\
- & \frac{1}{\sigma_{e_{n}^{2}}^{N}} \sum_{n=0}^{N-1}\left(P_{n \mid N}^{(p)}+\gamma^{2} P_{n-1 \mid N}^{(p)}-\gamma\left(P_{n, n-1 \mid N}^{(p)}+P_{n-1, n \mid N}^{(p)}\right)\right)(19) \\
- & \frac{1}{\sigma_{0}^{2}}\left|P_{0 \mid N}^{(p)}\right|^{2},
\end{aligned}
$$

with $P_{n \mid N}^{(p)} \stackrel{\text { def }}{=} \mathrm{E}\left(\alpha_{n} \alpha_{n}^{*} \mid \mathbf{y}, \mathbf{a} ; \tau^{(p)}\right), P_{n, n-1 \mid N}^{(p)} \stackrel{\text { def }}{=} \mathrm{E}\left(\alpha_{n} \alpha_{n-1}^{*} \mid \mathbf{y}\right.$, $\left.\mathbf{a} ; \tau^{(p)}\right), P_{n-1, n \mid N}^{(p)} \stackrel{\text { def }}{=} \mathrm{E}\left(\alpha_{n-1} \alpha_{n}^{*} \mid \mathbf{y}, \mathbf{a} ; \tau^{(p)}\right)$ and $\hat{\alpha}_{n \mid N}^{(p)} \stackrel{\text { def }}{=}$ 
$\mathrm{E}\left(\alpha_{n} \mid \mathbf{y}, \mathbf{a} ; \tau^{(p)}\right)$. We remark that the terms given by (20), (19) and the variance-dependent constant $C$ do not depend on $\tau$, then these terms can be removed from (18).

Since

$$
\begin{aligned}
S_{n \mid N}^{(p)} & \stackrel{\text { def }}{=} \mathrm{E}\left(\left|\alpha(n)-\hat{\alpha}_{n \mid N}^{(p)}\right|^{2} \mid \mathbf{y}, \mathbf{a} ; \tau^{(p)}\right) \\
& =P_{n \mid N}^{(p)}-\hat{\alpha}_{n \mid N}^{(p)} \hat{\alpha}_{n \mid N}^{(p) *}
\end{aligned}
$$

By deducing the value of $\hat{P}_{n \mid N}^{(p)}$ from the relation (21) and replacing it into (18), we obtain (13).

\section{B. REFERENCES}

[1] T. Aulin and C. E. Sundberg, "Continuous phase modulationparts I and II," IEEE Trans. Commun., vol. COM-29, pp. 196225, Mar. 1981.

[2] P. Chevalier and F. Pipon, "New insights into optimal widely linear array receivers for the demodulation of BPSK, MSK, and GMSK signals corrupted by noncircular interferencesapplication to SAIC," IEEE Trans. Signal Process., vol. 54, no. 3, pp. 870-883, 2006.

[3] H. Abeida and J.P. Delmas, "Statistical performance of MUSIC-like algorithms in resolving noncircular sources," IEEE Trans. Signal Process., vol. 56, no. 9, pp. 4317-4329, September 2008.

[4] U. Mengali and A. N. D'Andrea, Synchronization techniques for digital receivers, New York: Plenum Press, 1997.

[5] T. Aulin and C.-E. Sundberg, "Synchronization properties of continuous phase modulation", in Proc. Globcom, pp. D7.1.1D7.1.7., Nov. 1982.

[6] J. Huber and W. Liu, "Data-aided synchronization of coherent CPM receivers," IEEE Trans. Commun., vol. 40, no. 1, pp. 178-188, Jan. 1992.

[7] J. Riba and G. Vazquez, "Non-data-aided frequency offset and symbol timing estimation for binary CPM: performance bounds," in Proc. ICASSP, 2000, vol. 5, pp. 2549-2552.

[8] A. N. D'Andrea and U. Mengali, "Symbol timing estimation with CPM modulation," IEEE Trans. Commun., vol. 44, no. 10, pp. 1362-1372, Oct. 1996.

[9] M. Morelli and U. Mengali, "Joint frequency and timing recovery for MSK-type modulation," IEEE Trans. Commun., vol. 47, no. 6, pp. 938-946, Jun. 1999.

[10] H.Wang and P. Chang, "On verifying the first-order Markovian assumption for a Rayleigh fading channel model," IEEE Trans. Veh. Technol., vol. 45, pp. 353-357, May 1996.

[11] R. E. Kalman, "A new approach to linear filtering and prediction problems," Transactions of the ASME, Journal of Basic Engineering, vol. 82, pp. 34-45, March 1960.

[12] A. P. Dempster, N. M. Laird and D. B. Rubin, "Maximum likelihood from incompleter data via EM algorithm," J. Roy. Statist. Soc., vol. 39, pp. 1, 1998.

[13] R. Dabora, J. Goldberg, and H. Messer, "Training-Based timedelay estimation for CPM signals over time-selective fading Channels," IEEE Trans. Commun., vol. 52, no. 7, pp. 11691177, July 2004.
[14] V. Digalakis, J. Rohlicek and M. Ostendorf, "ML estimation of a stochastic linear system with the EM algorithm and its application to speech recognition," IEEE Trans. Speech Audio Processing, vol. 1, no. 4, pp. 431-442, Sept. 1993.

[15] M. E. Khan and D.N. Dutt, "An expectation-maximization algorithm based kalman smoother approach for event-related desynchronization (ERD) estimation from EEG," IEEE Trans. Biom., vol. 54, no. 7, pp. 1191-1198, July 2007.

[16] J. F. G. de Freitas, M. Niranjan and A.H. Gee, "Nonlinear state space learning with EM and neural networks," IEEE International Workshop on Neural Networks in Signal Processing, Cambridge, England. pp. 254-263, Sept. 1998.

[17] H. Chen, R. Perry and K. Buckley, "Direct and EM-based MAP sequence estimation with unknown time-varying channels," in Proc. IEEE Int. Conf. Acoust., Speech, Signal Processing, vol. 4, pp. 2129-2132, May 2001.

[18] F. Patenaude, J. H. Lodge and P.A. Galko, "symbol timing tracking for continous phase modulation over fast flat-fading channels," IEEE Trans. veh. Techn., vol. 40, pp. 615-626, August 1991.

[19] P. Tichavsky , C. H. Muravchik and A. Nehorai, "Posterior Cramér Rao bounds for discrete-time nonlinear filtering," IEEE Trans. Signal Process., vol. 46, pp. 1386, May 1998.

[20] C. H. Knapp and G. C. Carter, "The generalized correlation method for estimation of time delay," IEEE Trans. Acoust., Speech, Signal Processing, vol. ASSP-24, pp. 320-327, Aug. 1976.

[21] W. C. Jakes, Microwave Mobile Communications, New York: Wiley, 1974.

[22] K. E. Baddour and N. C. Beaulieu, "Autoregressive modeling for fading channel simulation," IEEE Trans. Wireless Commun., vol. 4, no. 4, pp. 1650-1662, Jul. 2005.

[23] H. E. Rauch, "Soltutions to the linear smoothing problem," IEEE Trans. on Automatic Control, vol. 8, pp. 371-372, 1963.

[24] H. L. Van Trees, Detection, Estimation, and Modulation Theory, Part I, New York, NY: John Wiley and Sons, 1968.

[25] H. Abeida, J. M. Brossier and L. Ros, "Time-varying multipath channel estimation for CPM signals using the EM algorithm," in prepartion to IEEE Trans. on Signal Processing

[26] Y. Rockah and P.M. Schultheiss, "Array Shape Calibration Using Sources in Unknown Locations-Part I: Near-Field Sources," IEEE Trans. Acoust., Speech, Signal Process, vol. ASSP-35, pp. 286-299, March 1987.

[27] S. Bay, B. Geller, A. Renaux, J.P. Barbot and J.M. Brossier, "On the Hybrid Cramer-Rao bound and its application to dynamical phase estimation," IEEE Signal Processing letters, vol. 15, pp. 453-456, 2008.

[28] H. Abeida and J.P. Delmas, "Cramer-Rao bound for direction estimation of non-circular signals in unknown noise fields," IEEE Trans. on Signal Processing, vol. 53, no. 12, pp. 46104618, December 2005.

[29] H. Cramér, mathematical methods of statistics, Princeton, NJ: Prinston Univetisty press, 1946. 